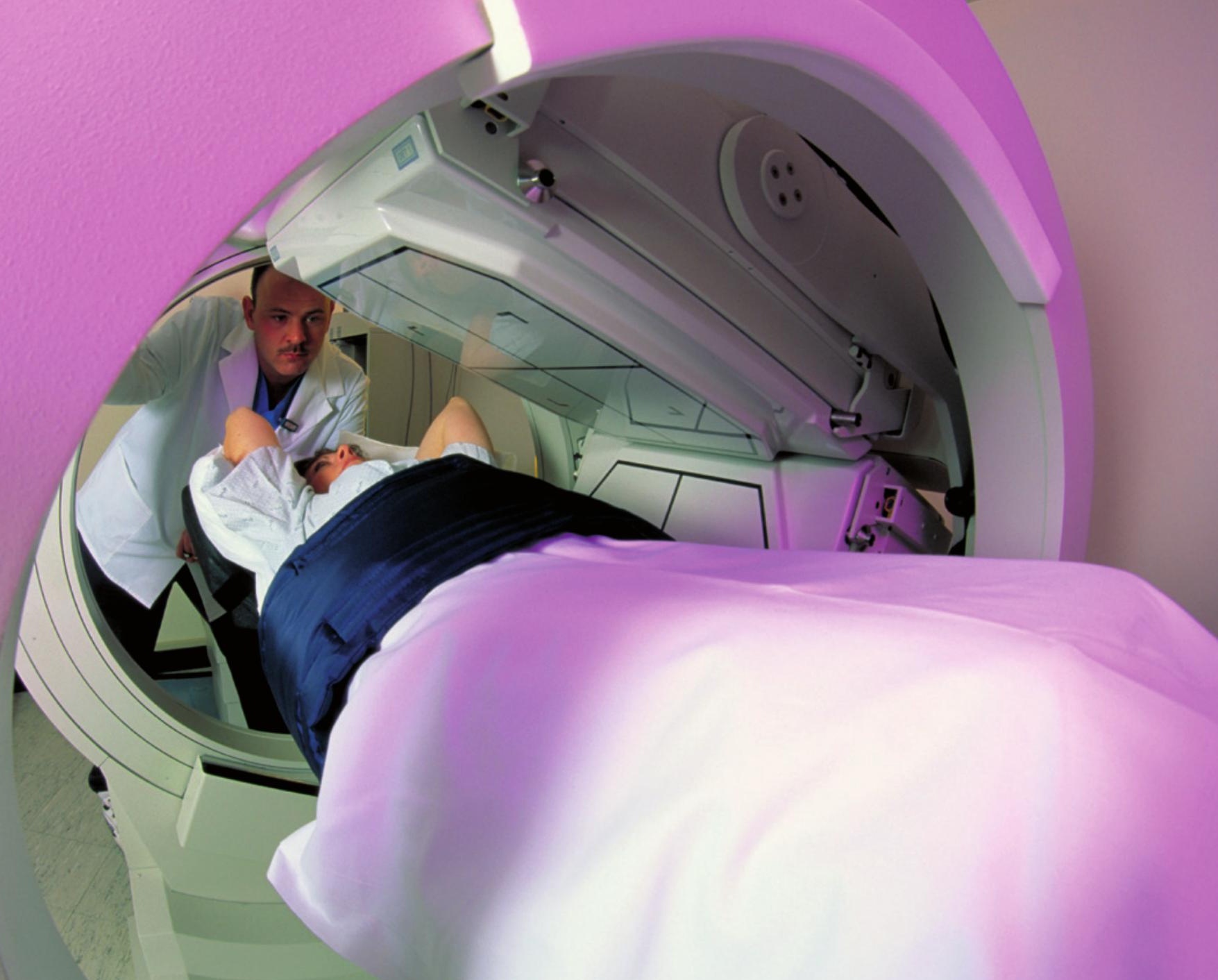

\title{
THE MEDICAL TESTING CRISIS
}

\section{With a serious shortage of medical isotopes looming, innovative companies are exploring ways to make them without nuclear reactors.}

\author{
BY RICHARD VAN NOORDEN
}

n 2009, two nuclear research reactors shut down for repairs and maintenance. This was not surprising, given that both were around half a century old. But these reactors happened to produce most of the world's supply of the radioactive tracer technetium-99m, an isotope injected into patients in 70,000 diagnostic scans a day. Hospitals around the world went into a panic.

Finding themselves suddenly short of the crucial isotope, doctors cancelled scans, postponed operations or switched to older diagnostic techniques that exposed patients to higher doses of radiation. "It was the isotope equivalent of an electricity blackout," says Ronald Schram, who manages one of the affected reactors, the High Flux Reactor at Petten in the Netherlands. Nobody knows exactly how much damage was done, says Fred Verzijlbergen, head of the department of nuclear medicine at Erasmus Medical Center in Rotterdam, but "it was very serious. Many hospitals didn't receive technetium for weeks."

The crash made it painfully clear that the world's medical-isotope supply chain was dangerously fragile, relying heavily on about four government-subsidized reactors built in the 1950s and 1960s. Isotope supplies have taken a hit again and again, most recently last month, when Canada's Chalk River reactor shut down unexpectedly for a few days at the same time as two other reactors. And more shortages are coming. The Chalk River reactor, which produces close to one-third of current global supplies, is slated to end production of isotopes in 2016.

But for nuclear engineer Greg Piefer, the crisis presents an opportunity. In 2005, fresh out of a nuclear-engineering doctorate at the University of Wisconsin-Madison, he had dreamed up a way to use particle accelerators - rather than nuclear reactors, with their problematic waste - to transform uranium into technetium. His idea did not get much attention at the time. After the 2009 disaster, however, politicians demanded new ways of making medical isotopes, particularly in the United States, which accounts for $50 \%$ of world medical-isotope demand but has no local production capacity. Piefer's ideas, and those of other aspiring entrepreneurs, were thrown into the spotlight.

\section{TECHNICAL CHALLENGE}

At least five North American companies and collaborations, including Piefer's firm, SHINE Medical Technologies in Madison, are pioneering methods that should produce medical isotopes in the next few years. It 
Many diagnostic scans rely on radioactive technetium-99m. is not clear which will win out - nor whether they can replace the conventional reactor approach or be ready soon enough to avert another shortage. "It's boiling down to a rather critical situation in 2015-16," says Schram.

Technetium-99m is often called the workhorse of modern medical imaging, because it accounts for about $80 \%$ of the world's use of radioactive isotopes in nuclear medicine, $90 \%$ of which is diagnostic scans. A $\gamma$-ray emitter with a half-life of just 6 hours, it can be attached to a molecule that targets the organ of interest. Medical scans known as single-photon emission computed tomography (SPECT) then pick up the glow of the radioisotope. Such tests are used to check how well blood is flowing to heart muscles, to spot whether cancers have spread through bones and to assess blood flow in the brain.

The creation of technetium-99m involves one of those miraculous, globe-crossing supply chains that modern economies have rendered commonplace. The journey starts with enriched uranium from the United States, which is made into plates and shipped to research reactors around the world. Each plate is baked for a week in the glare of a nuclear reactor's neutrons, which fission about $6 \%$ of the uranium into molybdenum- 99 . This has a half-life of 66 hours and slowly decays into technetium. Hospitals across the globe purchase 'moly cows' - paint-tin-sized devices that hold the molybdenum-99 bound tightly to alumina. By flushing the technetium out with saline solution, hospital technicians can milk the moly cows for fresh supplies of technetium for up to two weeks.

Nuclear reactors are the most efficient way to produce molybdenum, says Benard Ponsard, who manages the isotope-producing BR2 reac-

tor in Mol, Belgium. Many others agree. In the 1990s, Canada planned

new reactors that would have circumvented many of the shortages, but these were mothballed in 2008 after technical problems emerged that proved too expensive to fix. So other countries are now racing to fill the gap. BR2 aims to start upgrading its medical-isotope capacity at the end of 2014. The OPAL reactor in South Sydney, Australia, is planning upgrades that would quadruple its isotope supplies by 2017. New reactors or upgrades are planned from Argentina to China (see 'Supply fix').

This promises a lot of production capacity. But there could still be problems. The new reactors might not supply enough isotopes to provide a sufficient cushion in case of major breakdowns, notes Robert Atcher, director of the US National Isotope Development Center, created in 2009 by the Department of Energy to help to manage isotope distribution.

\section{PRICE HIKES}

More crucially, the cost of reactor-sourced molybdenum could skyrocket. Because the reactors are involved in research, they are subsidized by their host governments and sell their molybdenum at below-market prices. This means there is little incentive for companies to invest in new production facilities, concluded a post-crisis review by the Nuclear Energy Agency (NEA) of the Organisation for Economic Co-operation and Development (OECD) in Paris. The NEA has endorsed a plan to end the subsidies, which those in the supply chain are now preparing for. According to Ron Cameron, head of nuclear development for the agency, the price of molybdenum from reactors could increase as much as sevenfold when that happens. Meanwhile, the United States has decided to stop exporting highly enriched uranium, because it might be intercepted to make nuclear weapons. By 2020, reactors will have to make do with low-enriched uranium fuel and plates - which could increase molybdenum costs from reactors by another $40 \%$. The final price to the middle-man is "very much up in the air", says Atcher, who guesses it might increase 15-fold.

That has led some to seek a radical alternative. Rather than depending on a few distant centres of production, each costing hundreds of millions of dollars, hospitals could get their isotopes locally from facilities that have small medical cyclotrons costing just a few million dollars, says Paul Schaffer, head of the nuclear medicine division at TRIUMF, Canada's national laboratory for particle and nuclear physics in Vancouver, and leader of a team pursuing this idea.

In the cyclotron model, neither nuclear reactors nor uranium are needed. A beam of accelerated protons shoots into a target of molybdenum-100, creating technetium-99m directly. Technetium's 6-hour halflife means that the product cannot be transported far: a single cyclotron could cover maybe a 400-kilometre radius. The idea is to have lots of cyclotrons distributed across major urban areas. That is not as ambitious as it might seem, points out TRIUMF spokesman Tim Meyer: many hospitals already use in-house cyclotrons to produce isotopes for a more advanced form of imaging, positron emission tomography.

In June, the TRIUMF team announced that by running an upgraded cyclotron in Vancouver overnight, it could make enough technetium to satisfy the city's needs. "The dozen or so cyclotrons [already] in Canada could cover $90 \%$ of the population and $50 \%$ of the geography" when adapted with a TRIUMF upgrade kit, says Meyer. The team awaits approval from Health Canada, which will confirm that its technetium is safe for use. Schaffer says that cyclotrons will not produce enough technetium to supply all of Canada's needs by 2016. "But a decentralized supply is certainly possible in the long term," he says.

Other countries seem to be interested in the strategy. Advanced Cyclotron Systems - a firm in Richmond, Canada, that sells cyclotrons and is working to make cyclotron technetium - has had inquiries from the United Kingdom, Saudi Arabia, Thailand and more. "It's got health authorities in many countries quite excited," says John Taylor-Wilson, the company's vicepresident of marketing and business development.

Atcher, however, is not convinced that the approach will be helpful in the United States, where, by chance, most hospitals have lower-power cyclotrons that could not produce as much technetium. And if a cyclotron goes down for repair, an urban area might be left without a back-up plan.

\section{BRIGHT IDEAS}

Piefer has a plan that is a little less radical than the cyclotron model. His team at SHINE wants to stick with the current distribution system, but get rid of the expensive nuclear reactor at the system's heart.

SHINE's technology uses a linear accelerator to slam deuterium ions into tritium gas, producing helium and neutrons. The neutron flux is orders of magnitude less than that emerging from a nuclear reactor. Instead of a small uranium plate, the neutrons are fired into a couple of hundred litres of warm, low-enriched uranium salts. The molybdenum can be rinsed away using ion-exchange resins, and the unconverted uranium recycled for use in the same facility. Nuclear waste is produced from the uranium target, but it is only a small fraction of that produced from reactor fuel, notes Piefer.

Piefer hopes to build a facility in Janesville, Wisconsin, which he says would supply half of the United States' need for technetium (about onequarter of world demand). But construction has not started yet, because the firm - like any US company working with uranium — has to wait for a permit from the Nuclear Regulatory Commission. And it needs to raise another US\$150 million, on top of $\$ 30$ million raised so far from a Department of Energy grant and investors. "If sufficient funding were available, we'd start production by the end of 2016," Piefer says - about the $\rightarrow$ NATURE.COM For a podcast about alternative isotope technologies, see: go.nature.com/w74z5a same time the Chalk River reactor ends its supply.

Piefer will face some competition from right across town. Madison is home to another innovative medical-isotope company: NorthStar, which also hopes to supply half the United States' medical isotope needs in the next few years - and in the 


\section{SUPPLY FIX}

Today, just a few nuclear reactors provide global supplies of technetium-99m (Tc-99), the most popular tracer used in medical scans. Many of the reactors are old and some will soon stop producing medical isotopes, leaving the world vulnerable to shortages.

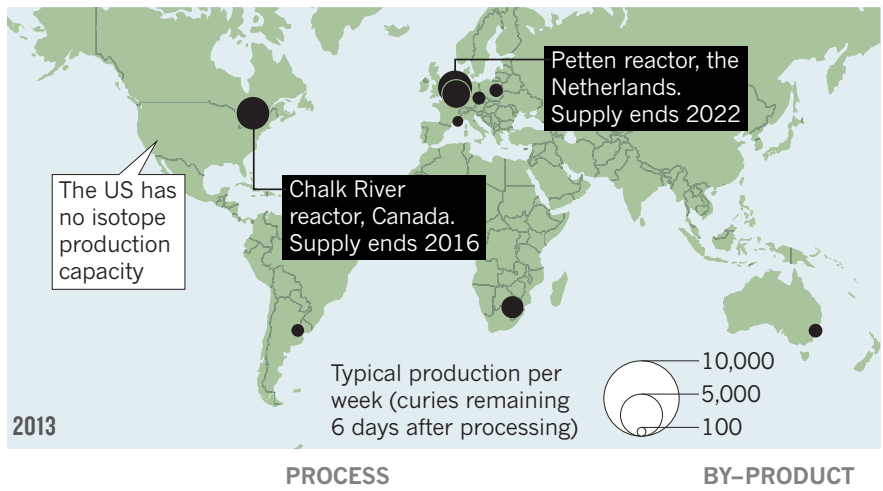

NUCLEAR REACTOR

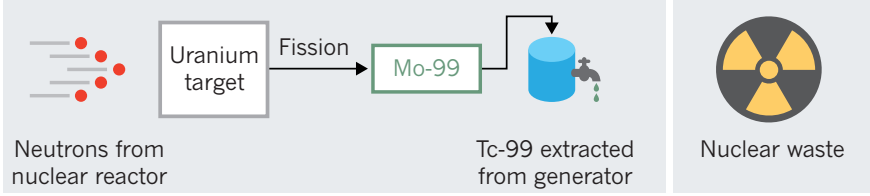

New nuclear reactors will diversify isotope supplies in coming years. But researchers are also developing production methods that avoid using a reactor or a uranium target. Some schemes produce little or no nuclear waste.

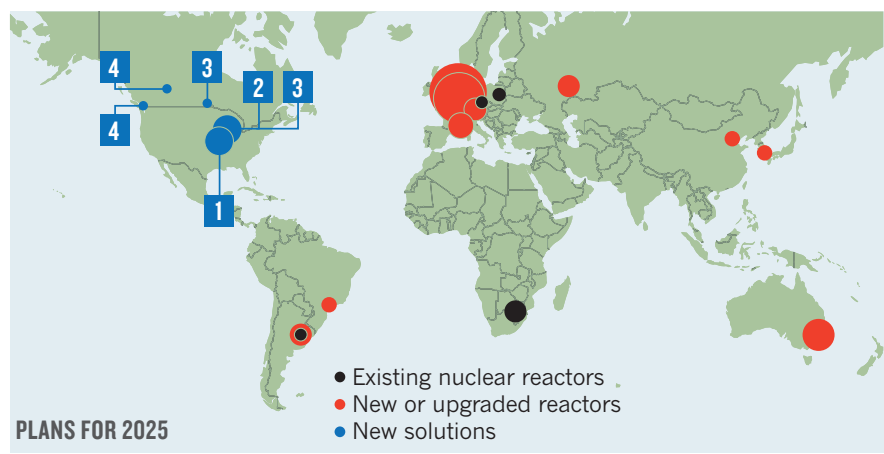

REACTOR WITH NEW TARGET (NORTHSTAR)

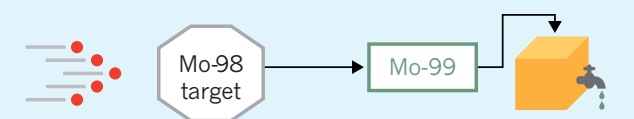

Neutrons from nuclear reactor
Tc-99 extracted from new generator

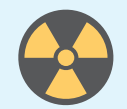

Nuclear waste
ACCELERATOR (SHINE MEDICAL TECHNOLOGIES)

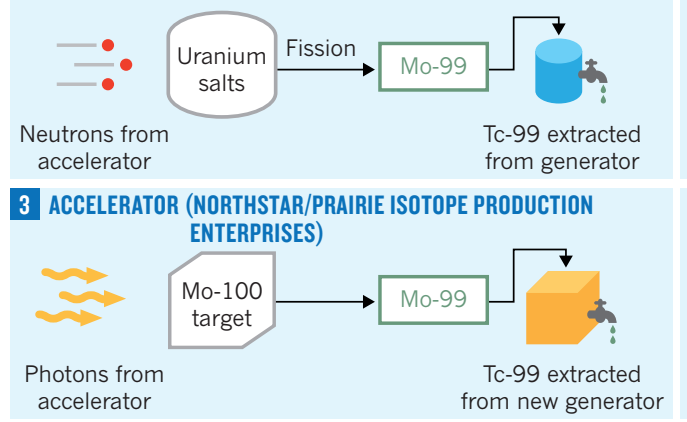

$\theta$

Nuclear waste

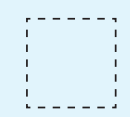

No nuclear waste

\section{from new generator}

4 CYCLOTRON (TRIUMF/ADVANCED CYCLOTRON SYSTEMS

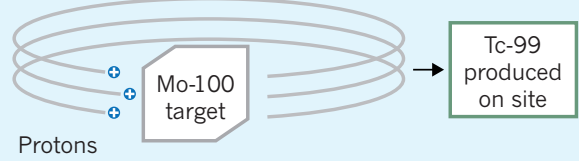

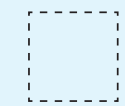

No nuclear waste longer term, aims to double that output with a second project. The fact that it has ended up so close to SHINE is "a bizarre coincidence", says Piefer; the rivals are "cordial but competitive", he adds.

Whereas SHINE avoids using a nuclear reactor but keeps a uranium target, NorthStar aims to do the opposite. It ditches the uranium target but — at least at first — still makes use of a reactor. The company's shortterm plan is to use the research nuclear reactor at the University of Missouri in Columbia to fire neutrons into a molybdenum-98 target, making molybdenum-99. This would be very quick to get up and running, but it invites complications on another front. Rather than being able to separate molybdenum-99 from the surrounding uranium, which is relatively easy, the company has the trickier task of separating the desired isotope from the molybdenum starting material. So NorthStar has had to design a generator to replace the moly cow. The result, about the size of a microwave oven, requires a computer, pipes and valves to extract technetium - more complex than the simple saline wash that hospitals use today.

In March, NorthStar submitted a New Drug Application to the US Food and Drug Administration (FDA), seeking to prove that its milked technetium is equivalent to what comes out of moly cow generators. It is hoping to receive the final word by the end of the year, says James Harvey, the company's chief science officer. "The Missouri project will be fully in production by mid-2014," Harvey says.

In the long-term, NorthStar has a more ambitious plan that would again cut reactors out of the system. It plans to use high-energy photons from a linear accelerator to kick neutrons out of molybdenum-100 to produce molybdenum-99. (Prairie Isotope Production Enterprises, a non-profit firm based in Winnipeg, Canada, is looking at a similar system, but on a smaller scale.) Again, NorthStar would need to use its microwave-sized hospital generator, assuming that it gets FDA approval. And although the company has raised $\$ 50$ million already, it will need much more to get this scheme off the ground: Harvey will not reveal a figure, but says it is a lot less than $\$ 150$ million.

Atcher has doubts about both NorthStar and SHINE. "In a nutshell, both of these companies are start-up companies," he says, adding, "2016 is not that far away and they are scrambling to get their programmes going."

\section{MARKET FORCES}

Underlying all the jostling are questions to which no one has good answers: how expensive the technetium from these new technologies will be, and whether the schemes will create enough to replace the nuclearreactor approach. "Obviously, each of these competitors has a secret recipe where they think they can beat the odds," says Meyer. But the uncertainty in the economics scared away larger companies such as General Electric and Babcock and Wilcox, both of which initially showed an interest in developing medical-isotope schemes but backed out last year.

Atcher thinks that reactors will always come out ahead. Others see a more diverse future. "The long-term scenario will really be driven by the market," says Schaffer. "I equate it to the electricity market, where we have nuclear, wind, hydroelectric, solar and so on. And the price of that source of electricity pretty much defines its share of the market. I believe the same thing will happen with isotopes, with sources from cyclotrons, linear accelerators and nuclear reactors."

So can hospitals avert catastrophe in 2016, when the Chalk River production facility shuts down? "On paper, it looks like the world can compensate," says Schaffer. But, he adds "it's such a dynamic situation". Cameron, more soberly, says that the answer will not be clear until he sees which reactors and companies ramp up production. "We have to do a lot of sums to see how the effects will balance out."

All the uncertainty about the technologies is leaving doctors such as Verzijlbergen concerned. "There is a lot of optimism but we need proof," he says. "From the medical side I am interested in reliable supply." He still worries that in a few years he will have to go to patients with difficult news: that a widespread shortage of technetium means that they cannot have the diagnostic tests that they need.

Richard Van Noorden is a senior reporter at Nature in London. 